\title{
Pyogenic osteomyelitis of the spine in the elderly: three cases of a synchronous non-axial infection by a different pathogen
}

\author{
GJ Velan*,1, J Leitner ${ }^{1}$ and R Gepstein ${ }^{1}$ \\ ${ }^{1}$ Spine Care Unit, Meir Hospital, Kfar-Saba, Israel
}

\begin{abstract}
Study design: A retrospective chart review of patients over 65 years of age treated at the Spine Care Unit for pyogenic vertebral osteomyelitis.

Objectives: To assess the reliability of peripheral blood, urine and sputum cultures in the treatment of pyogenic vertebral osteomyelitis in the elderly.

Setting: Study performed at the Spine Care Unit, Meir Hospital, Kfar-Saba, Israel.

Methods: The Meir Hospital records were searched for patients over 65 years of age, treated at the Spine Care Unit for pyogenic vertebral osteomyelitis. Charts, culture results and imaging studies were reviewed. A medline literature search was performed to survey the literature regarding pyogenic vertebral osteomyelitis in the elderly with emphasis on diagnostic imaging modalities and surgical treatment.

Results: Three patients were identified with concurrent peripheral infection by a different organism than the organism causing the vertebral osteomyelitis. Delay in correct diagnosis led to neurologic impairment in all patients and surgical treatment was performed in all three to drain the epidural abscess, decompress the spinal cord and obtain direct tissue culture. Following decompression and epidural abscess evacuation, one patient has functionally recovered and was ambulating with a cane, two patients did not recover and remained paraparetic and ambulate in a wheelchair.

Conclusions: Pyogenic vertebral osteomyelitis in the elderly can be caused by a different pathogen than that isolated from blood, sputum or urine cultures. In the elderly, a biopsy of the vertebral lesion should be obtained for susceptibility studies prior to conservative treatment with bracing and intravenous antibiotics.
\end{abstract}

Keywords: pyogenic vertebral osteomyelitis; elderly; diagnosis

\section{Introduction}

Pyogenic vertebral osteomyelitis has been established in the last decade as a major cause of morbidity in the elderly population. ${ }^{1,2}$ The treatment of pyogenic vertebral osteomyelitis in the absence of epidural space invasion is immobilization and intravenous antibiotics. $^{3}$ Often, organisms are cultured from peripheral blood, or to a lesser extent, other sterile sites as synovium, lower respiratory tract or urinary tract. In these circumstances, a direct vertebral aspirate or biopsy is not considered mandatory. ${ }^{4}$ In the elderly, comorbidity and high prevalence of genitourinary infections make concurrent infection a possibility. ${ }^{1,5,6}$ In one study, ${ }^{1} 18$ of 111 patients had a comorbid state adjacent to the site of infection, 44 of 111 had impaired immune status and urinary tract infection was the most common source of hematogenic spread to the spine. This study did not account for synchronous blood and urine cultures that were positive to an organism

*Correspondence: GJ Velan MD, Orthopaedic Surgery, Mayo Clinic, 200 First Street SW, Rochester, Minnesota, 55905 USA different than the one infecting the vertebra. Other authors ${ }^{5,6}$ recognized genitourinary tract infections as the primary infection site in 30 per cent of patients with vertebral osteomyelitis. These investigators did not study the possible concurrent pyogenic vertebral osteomyelitis and urinary tract infection by different microorganisms in the elderly patient.

We report three cases of pyogenic vertebral osteomyelitis in elderly patients. Inadequate antimicrobial therapy, based on cultures and susceptibility studies of peripheral blood, synovial fluid or lung abscess aspirate, resulted in neurological deterioration and consequent major spinal cord dysfunction.

\section{Illustrative cases}

Case 1

A 74-year-old woman presented with severe localized upper back pain without radiation, spastic gait disturbance but no impaired motor function. Four weeks previously the patient had a thoracotomy to drain an abscess in the right lung. Cultures of abscess 
aspirate were positive for Klebsiella bacilli, blood cultures taken at that time were sterile. Radiographic images taken at this time demonstrated destruction of T6 and T7 vertebral bodies. Based on the known respiratory infection and the advanced stage of vertebral destruction, a presumptive diagnosis of Klebsiella osteomyelitis was made. Antimicrobial therapy was directed accordingly. Though adequate antibiotic levels were achieved, patient did not improve, erythrocyte sedimentation rate (ESR) remained maximally elevated and after 2 weeks the patient was afflicted with acute T6 paraparesis. MRI revealed osteomyelitis of the T6 and T7 vertebral bodies with epidural extension and spinal cord compression. When the patient was operated, a direct tissue culture identified Staph. Aureus as the offending organism. Corpectomy of T6 and T7 was performed, fusion with fibular strut graft without instrumentation and TLSO was fitted. The patient was treated with intravenous Vancomycin for 6 weeks, ESR declined and by 3 months radiographic resolution and fusion were evident. After an intensive spinal cord injury rehabilitation program the patient remained paraparetic and a wheelchair ambulator.

\section{Case 2}

A 76-year-old farmer complained of back pain for 6 months, orthopaedic examination at that time and Xray of the lumbar spine have interpreted the source of his pain as degenerative changes. The patient complained of marked worsening of this pain, 2 weeks after the diagnosis of a Methycillin Resistant Staphylococcus Aureus (MRSA) septic arthritis of his left elbow. The patient was treated with intravenous Vancomycin but his back pain worsened and paraparesis evolved slowly. The patient was referred to our institution, at that time he was paraparetic at T8 level. MRI identified a lytic lesion in vertebral bodies D9 and D8 with epidural extension. Due to the epidural extension and manifest spinal cord compromise we elected to perform D8 and D9 corpectomy, anterior fusion with autogenous rib graft, and the patient was fitted with a TLSO. Brucella Melitensis was isolated from direct culture, obtained during surgery. At 3 months postoperatively the patient had a slightly elevated ESR, no local tenderness, mild T8 paraparesis and ambulated in a wheelchair. Thoracic spine radiograph taken 6 months postoperatively showed solid anterior fusion without deformity.

\section{Case 3}

A 78-year-old female was hospitalized for 40 days due to septic arthritis of the her right knee. Klebsiella sp. was isolated from blood and synovial joint fluid cultures. The patient status deteriorated despite culture directed antimicrobial therapy with gentamycin and second-generation cephalosporin. At the 45th day, a previously mild back pain attributed to prolonged recumbence has worsened considerably. On physical examination D6 paraparesis was evident. Plain radiographs and CT scan showed marked destruction of D5 vertebral body. We elected to perform a D5 corpectomy and rib graft anterior fusion without instrumentation. Methycillin sensitive Staph. aureus sp. was isolated from vertebral biopsy. The patient was treated with intravenous Methycillin for 6 weeks. She had recovered full motor function and at the 1 year follow up was ambulating independently with a cane.

\section{Discussion}

The incidence of vertebral osteomyelitis has risen in the last decades, ${ }^{1,2,7}$ mostly among the elderly and immunocompromised. In patients without predisposing surgery or paraspinal infection, spread of the pathogen is through the hematogenous route from a peripheral focus. ${ }^{8}$ Hematogenous seeding, following genitourinary tract intervention, ${ }^{1}$ skin infection and blunt trauma to the back $^{5}$ are known pathogenic mechanisms. Though Staphylococcus Aureus is the common pathogen in the general population, ${ }^{9}$ E.Coli and Proteus spp. are more likely pathogens in the elderly, ${ }^{5,6}$ due to seeding from the genitourinary tract. It has recently been established by Carragee ${ }^{1}$ that those over 65 years of age have a predilection towards low virulence organisms. Paralysis following pyogenic vertebral osteomyelitis is more common in those older than 65 years of age. ${ }^{3,6,10}$ Therefore, it is crucial to identify the offending organism correctly, at an early stage.

Medical treatment is efficacious if epidural spread and paraplegia did not occur. ${ }^{11,7}$ Early correct diagnosis can prevent major neurological disability. When the typical clinical picture is combined with radiological evidence of vertebral destruction and elevated ESR, a positive peripheral culture can be misleading in the elderly. A high prevalence of comorbid conditions and synchronous foci of infection in the elderly with different organisms, can lead to erroneous treatment decisions.

Open drainage of vertebral osteomyelitis is mandatory when epidural extension causes cord compression and paraplegia. ${ }^{4,8}$ In the absence of neurological deficit, most cases of vertebral osteomyelitis can be treated by immobilization and antimicrobial therapy. $7,9,11,12$ Successful conservative treatment in the young or adult patient is based upon correct diagnosis of the infecting organism. This can be made according to positive blood or urine cultures. ${ }^{10,13}$ If CT guided biopsy of the involved vertebra is omitted and treatment is directed according to peripheral source cultures, a close follow up should be exercised. Attention to the patient response to antimicrobial therapy is mandatory. Decline of ESR during the first month of conservative treatment proved to be a good prognostic sign, ${ }^{14}$ and can guide preliminary treatment. 
In the elderly patient we believe this approach is not warranted. The elderly, who have a high prevalence of genitourinary and skin infections, the potential to develop multiple sources for hematogenous spread to the vertebrae, makes the diagnosis of the organism through blood or urine culture not reliable.

The cases described here are rare, case no. 2 being peculiar to the Mediterranean basin where brucellosis is endemic and should be entertained in every case with exposure to sheep or cattle. Though the positive predictive value of urine or synovial fluid cultures is inferior to that of blood culture, the treating physicians in all three cases began treatment according to them. It was the neurological deterioration that alerted them to the possibility of misdiagnosis. Paraplegia indicated drainage and decompression of the spinal cord, while allowing the procurement of direct culture material.

In the elderly patient, a direct tissue culture should be obtained prior to commencement of antimicrobial therapy. CT guided needle biopsy is a safe and effective method, ${ }^{11-13,15-17}$ to obtain direct material for culture. When negative, an open biopsy through a thoracotomy or retroperitoneal approach can be performed if the tentative antimicrobial therapy is not effective, as demonstrated by persistent elevated ESR or extension of the abscess into the epidural space.

\section{References}

1 Carragee EJ. Pyogenic vertebral osteomyelitis. J Bone Joint Surg Am 1997; 79: 874-880.
2 Patzakis MJ et al. Analysis of 61 cases of vertebral osteomyelitis. Clin Orthor 1991; 264: 178 - 183.

3 Eismont FJ et al. Pyogenic and fungal vertebral osteomyelitis with paralysis. J Bone Joint Surg 1983; 65A: 19-29.

4 Gepstein R et al. Management of pyogenic vertebral osteomyelitis with spinal cord compression in the elderly. Paraplegia 1992; 30: $795-798$.

5 Sapico FL, Montgomerie JZ. Pyogenic vertebral osteomyelitis: report of 9 cases and review of the literature. Rev Infect Dis 1980; 2: $196-206$.

6 Sapico FL, Montgomerie JZ. Vertebral osteomyelitis. Infect Dis Clin North Am 1990; 4: 539 - 550.

7 Silverthorn KG, Gillespie WJ. Pyogenic spinal osteomyelitis: a review of 61 cases. Aus NZ Med J 1986; 99: 62-65.

8 Wiley AM, Trueta $\mathbf{J}$. The vascular anatomy of the spine and its relationship to pyogenic vertebral osteomyelitis. J Bone Joint Surg 1959; 41B: $796-809$.

9 Sapico FL. Microbiology and antimicrobial therapy of spinal infections. Orthop Clin North Am 1996; 27: 9-13.

10 Sonu C, Carragee E. Pyogenic vertebral osteomyelitis. Complic Orthop 1994; 9: 22 - 27.

11 Obana WG, Rosenblum ML. Non operative treatment of surgical infections. Neurosurg Clin North Am 1992; 3: 359-373.

12 Wisnesky RJ. Infectious diseases of the spine. Diagnostic and treatment considerations. Orthop Clin North Am 1991; 22: 491 501 .

13 Waldvogel RA, Vasey H. Osteomyelitis: the past decade. $N E n g J$ Med 1980; 303: $360-370$.

14 Carragee EJ, Kim D, van der Vlugt T, Vittum D. The clinical use of erthrocyte sedimenttion rate in pyogenic vertebral osteomyelitis. Spine 1997; 22: $2089-2093$.

15 Bateman JL, Pevzner MM. Spinal osteomyelitis: a review of 10 years' experience. Orthopedics 1995; 18: $561-565$

16 Stocker DJ, Kissim CM. Percutaneous vertebral biopsy: a review of 135 cases. Clin Radiol 1985; 33: 569-577.

17 Bender CE, Berquist TH, Wold LE. Imaging assisted percutaneous biopsy of the throacic spine. Mayo Clin Proc 1986; 61: $942-950$. 